\title{
Phase Shift Mask Fabrication by Laser Microlens Array Lithography for Periodic Nanostructures Patterning
}

\author{
Zhiqiang Huang ${ }^{* 1, * 2, * 3}$, Qun Ying Lin ${ }^{* 3}$ and Minghui Hong ${ }^{* 1, * 2}$ \\ ${ }^{* 1}$ Department of Electrical \& Computer Engineering, National University of Singapore, \\ Singapore 117576 \\ E-mail: elehmh@nus.edu.sg \\ ${ }^{* 2}$ Data Storage Institute, 5 Engineering Drive 1, Singapore 117608 \\ ${ }^{* 3}$ GLOBALFOUNDRIES SINGAPORE Pte Ltd, 60 Woodlands Industrial Park D, Street Two, \\ Singapore 738406
}

\begin{abstract}
Laser microlens array (MLA) lithography was used to fabricate arbitrary periodic array of patterns on photoresist. Reactive ion etching (RIE) was then utilized to etch and transfer the patterns down to the fused silica substrate. By controlling the etching process, the etched surface was $180^{\circ}$ out of phase with the unetched surface. Subsequently, the patterns were used as a phase shift mask for UV exposure. Destructive interference occurred at the edges of the phase shift structures, resulting in regions of low light intensities at the edges. It formed periodic array of smaller patterns on the unexposed photoresist, with feature sizes around $290 \mathrm{~nm}$. Simulation verified the experimental result.

DOI:10.2961/jlmn.2010.03.0010
\end{abstract}

Keywords: Microlens array, lithography, parallel processing, multi-beam, phase shift mask, periodic structures.

\section{Introduction}

Periodic arrays of nanostructures are used in an increasingly wide spectrum of applications, such as chemical and bio-medicals analyses [1-3], microelectronics for the synthesis of nanowires and nanostructures [4-5], templates for ordered microspheres array [6] and electromagnetic wave investigation and manipulation [710]. These applications demand the capabilities to create arrays of nanopatterns flexibly and rapidly. Photolithography is a valuable tool to pattern and form the desired micro/nano-structures. However, it is also an expensive step in the micro/nano-fabrication process. This is due largely to the high cost of photolithography stepper, as well as the expensive mask sets. Alternative methods, such as direct write techniques like e-beam and focused ion beam lithography, are attractive methods. However, direct writing methods suffer from low throughput since it is a serial process. This makes it time consuming to fabricate large areas of patterns. Laser microlens array (MLA) lithography [10-11] provides a potential solution to these needs. A MLA focuses the incident light into multiple focused micro-light beams at its focal plane. Using these focused light beams, different arbitrary patterns can be directly written. Meanwhile, it is a parallel process due to multiple light beams writing simultaneously. This offers a hybrid solution to cost effective direct write lithography with parallel processing, and is particularly advantageous for patterning large array of repetitive designs for many different possible applications. In this work, MLA lithography was employed to design and pattern periodic structures uniformly over a large area of photoresist coated on fused silica. The patterned designs were further processed to form phase shift structures on the fused silica substrate. Finally, the phase shift structures were used as a mask for UV lithography to print and pattern periodic array to create smaller structures.

\section{Experimental}

To carry out the Laser MLA lithography, a $\sim 1 \times 1 \mathrm{~cm}$ MLA, with a focal length of $\sim 28 \mu \mathrm{m}$ and a period of 50 $\mu \mathrm{m}$, was held firmly in place to an adaptor. A vacuum pump connected to the adaptor provides the suction force. The adaptor was then mounted onto a mechanical arm. Figure 1 shows the experimental setup. The fused silica sample, coated with negative photoresist (Ma-N 1407 from Microchem) at a rotational speed of $\sim 6000 \mathrm{rpm}$ to obtain a thickness of $\sim 500 \mathrm{~nm}$. It was placed on a precision nanostage, and also held in place by the vacuum suction. The nanostage has 7 degrees of freedom, X-Y-Z, fine-Z, tilt and tip to level the sample with respect to the MLA, and theta to rotate the sample. $400 \mathrm{~nm}$ laser light from a $2^{\text {nd }}$ harmonic Ti: Sapphire laser (Tsunami, Spectra Physics) irradiated upon the MLA. The power of the laser was varied from 5 to $20 \mathrm{~mW}$. The initial beam beam is $\sim 3 \mathrm{~mm}$. After passing through the beam expander, the laser beam width increases to $\sim 15 \mathrm{~mm}$. The light intensity at the central area is uniform and large enough to cover the whole MLA surface. The nanostage controlled and brought the sample to the focal plane of the MLA, and moved with respect to the microlens array to carry out patterning. The MLA focused the incident light into numerous individual focused micro-light beams that wrote and exposed the photoresist as the nanostage moved. After obtaining the patterns, reactive ion etching (RIE) was used to etch and transfer the patterns onto the fused silica substrate. The etching parameters were monitored so that the etched depth was controlled to an optical path length difference of $\lambda / 2$, 
forming phase shift structures on the substrate. It was then used as a lithography mask for UV exposure.

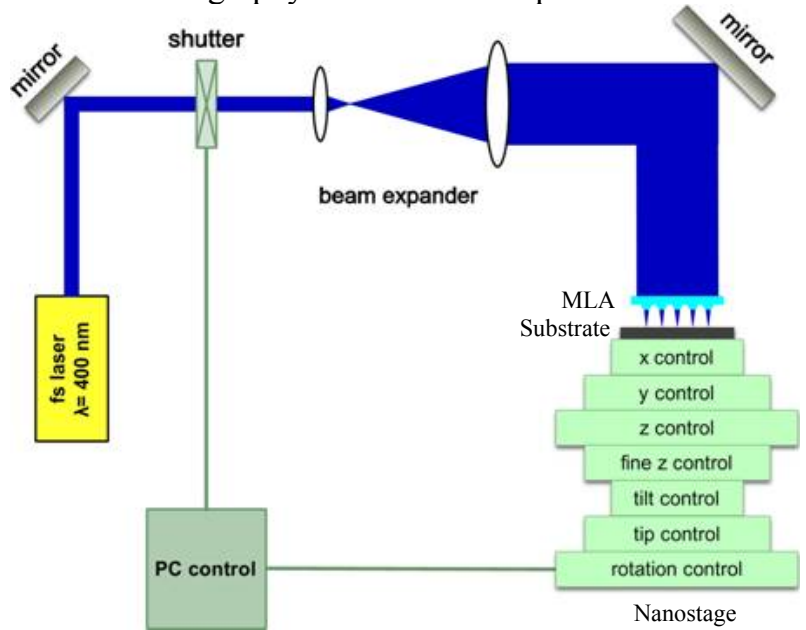

Fig. 1. Experimental setup for MLA lithography.

Figure 2 shows the process flow for transferring the patterns from the photoresist onto the fused silica. $50 \mathrm{~nm}$ of chromium was first coated onto a fused silica substrate, followed by a $20 \mathrm{~nm}$ thin layer of $\mathrm{SiO}_{2}$. Photoresist was then coated and patterned by the MLA lithography. RIE was used to etch and transfer the patterns onto the top 20 $\mathrm{nm}$ layer of $\mathrm{SiO}_{2}$, using $50 \mathrm{sccm}$ of $\mathrm{CF}_{4}$ as the etching gas, a chamber pressure of $20 \mathrm{mTorr}$, and a power of $250 \mathrm{~W}$. Chromium etchant was subsequently applied to etch the exposed areas of the chromium. A chromium hard mask layer was used to enable deep etching of the fused silica. After etching the chromium layer and cleaning in deionized water, RIE was used to etch the areas of fused silica which were not protected by chromium. Upon etching to the require depth, chromium etchant was applied to remove the remaining chromium.
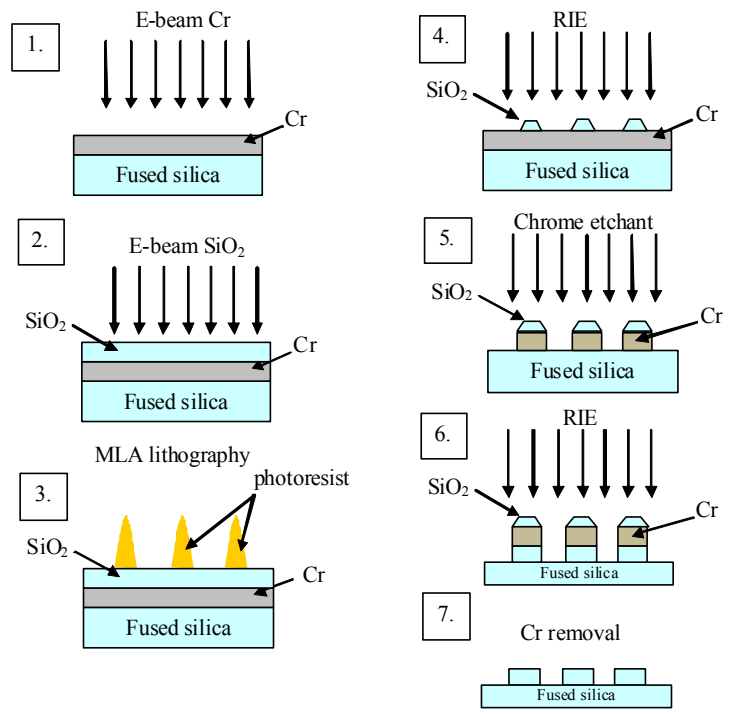

Fig. 2. Process flow to transfer the photoresist patterns onto the underlying fused silica substrate. $\mathrm{Cr}$ hard mask was implemented to enable deeper etching of the substrate.

\section{Results and discussion}

To achieve maskless direct-writing capabilities, computer numerical control (CNC) was used to control the movement of the nanostage during the laser MLA lithography. Various arbitrary shapes can be programmed and the shape paths would be traced accurately by the nanostage, allowing a variety of intricate designs to be fabricated by laser MLA direct writing. Figure 3a shows an example of a logo array designed and patterned using the laser MLA lithography technique. A circle with 3 diagonal lines was successfully produced on negative photoresist and transferred to fused silica substrate by RIE. Figure $3 \mathrm{~b}$ shows a generic IC design patterned using laser MLA lithography while the inset displays its $3 \mathrm{D}$ profile measured by AFM, illustrating that high quality patterns can be fabricated with this technique. The area size of the IC design is $10 \mu \mathrm{m} \times 10 \mu \mathrm{m}$ and it took about 12 seconds to complete the patterning when the nanostage moved at a speed of $5 \mu \mathrm{m} / \mathrm{s}$. These results demonstrated the flexibility and high throughput for its application in direct writing of large arrays of periodic structures.

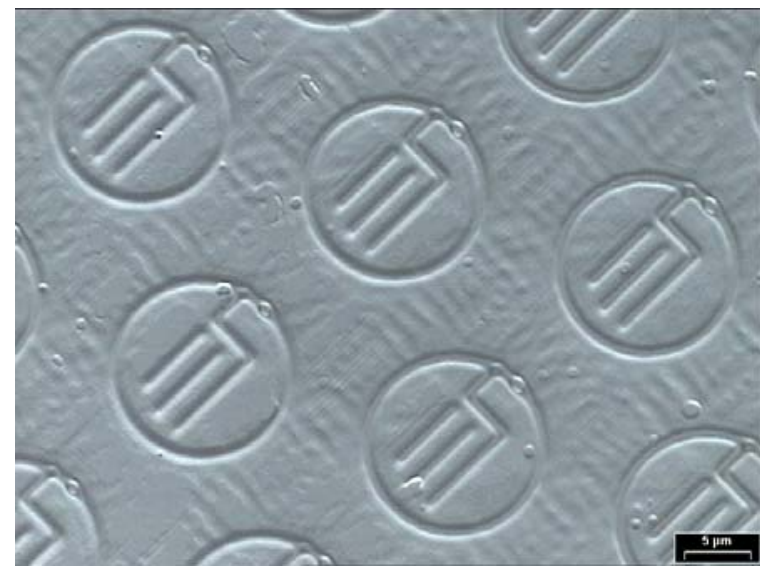

Fig. 3a. Logo array fabricated by laser MLA lithography.

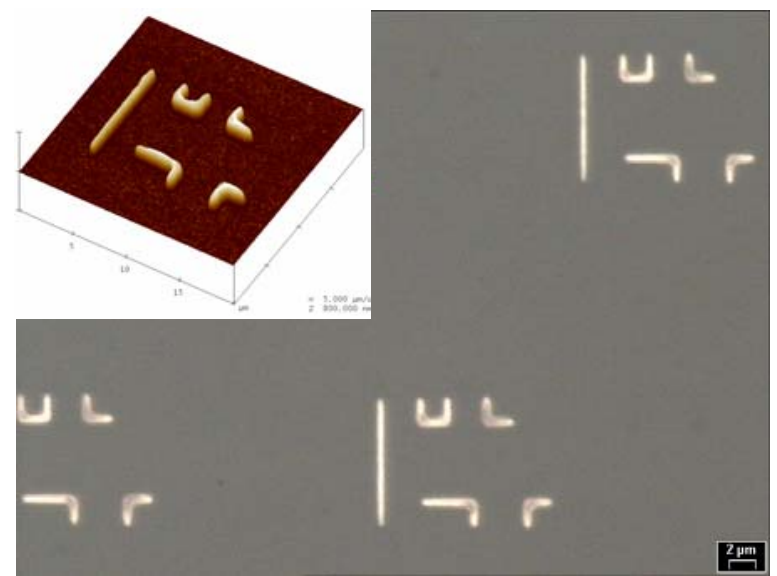

Fig. 3b. Generic IC design fabricated by Laser MLA lithography on fused silica. The inset shows the 3D AFM image of one unit cell. The shiny surface is due to the Cr hard mask.

Various studies [12-15] have shown that phase shift masks can be used to print smaller patterns. Laser MLA lithography, combined with RIE, was used as a cost 
effective method to fabricate periodic array of phase shift structures on fused silica. Subsequently, the phase shift structures were used as a mask for UV exposure of positive photoresist, forming arrays of patterns with smaller dimensions. Figure 4a shows an array of ' $L$ ' patterned by using the laser MLA lithography and transferred to the fused silica substrate by following the process illustrated in Fig. 2. The inset shows the 3D AFM image of the structure, illustrating the uniform height of the structure obtained through the use of a hard mask during RIE. This is crucial because the optimal phase shift was obtained when there is an optical path length difference of $\lambda / 2$ to introduce destructive interference. The phase shift structures were intended to be used for an illumination wavelength of $\lambda=365 \mathrm{~nm}$. The etched depth required is then $d=\lambda /[2(n-1)]$ $=385 \mathrm{~nm}$, where $\mathrm{n}$ is the refractive index of fused silica. The RIE process was carefully monitored to etch to the required depth. The ' $L$ ' shape array structured onto the fused silica surface is $100 \%$ transparent, with a width of $800 \mathrm{~nm}$. When light passes through the structure, the phase of the light is shifted out of phase by $180^{\circ}$. Destructive interference between the $0^{\circ}$ and the $180^{\circ}$ phase shifted light at the edges of the structure causes a steep drop in light intensity at the edges. Due to this effect, when the phase shift structures are used for UV exposure, areas at the phase edges do not expose the photoresist while other transparent areas transmit the incident light and expose the photoresist. Figure $4 \mathrm{~b}$ shows the result obtained by using the fabricated phase structures as a mask for UV exposure of positive photoresist. The UV light was from a mercury arc lamp ( $8 \mathrm{~mW}$ power, $\lambda=365 \mathrm{~nm})$, polarized in the $\mathrm{x}$ direction as indicated in Fig. 4c. 2 separate ' $L$ ' lines were left on the photoresist after development, which corresponded to the phase edges of the ' $L$ ' phase shift structure. The intensity minimum at the edges did not expose the positive photoresist, leaving the fine lines on the photoresist. The optical microscope measurement shows that the L lines have a dimension of $\sim 290 \mathrm{~nm}$, which is $\sim 3$ times smaller than the original L shape on the phase shift mask. By closer inspection, the inner L lines were broken at the perpendicular junction. To further study this result, theoretical simulation was carried out by Computer Simulation Technology (CST) software, which uses the Finite Integration Technique, simulating a similar L shape phase shift structure with the same material properties. Figure 4c plots the simulated light intensity below the phase shift structure upon the irradiation of $365 \mathrm{~nm}$ light. There are two regions of intensity minimum, as highlighted in the figure with dotted lines. These regions occur at the left and right edges of the phase shift structure, due to the destructive interference of light. Furthermore, at the right angle corner of the L shape, there is a coupling of light to the external light waves. This caused the right interior $\mathrm{L}$ line to be exposed and dis-jointed at the area near the corner. The theoretical result matches with the experimental result shown in Fig. 4b, where regions of intensity minimum did not expose the positive photoresist at the phase edges, and the right $\mathrm{L}$ was exposed at the right angle junction.

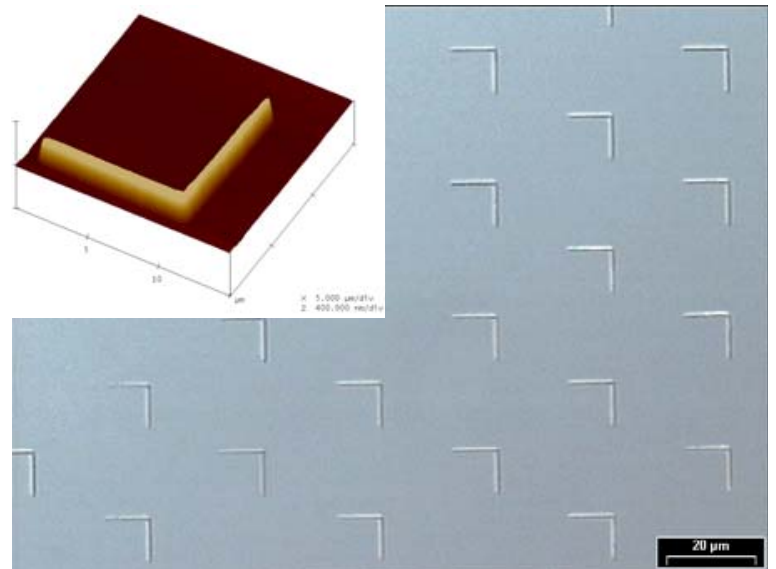

Fig. 4a Array of L patterned by laser MLA lithography and transferred to fused silica by RIE. Inset shows the 3D AFM image of the pattern. The surface is level, showing good control of the height uniformity, which is crucial for destructive interference to occur at the edges.

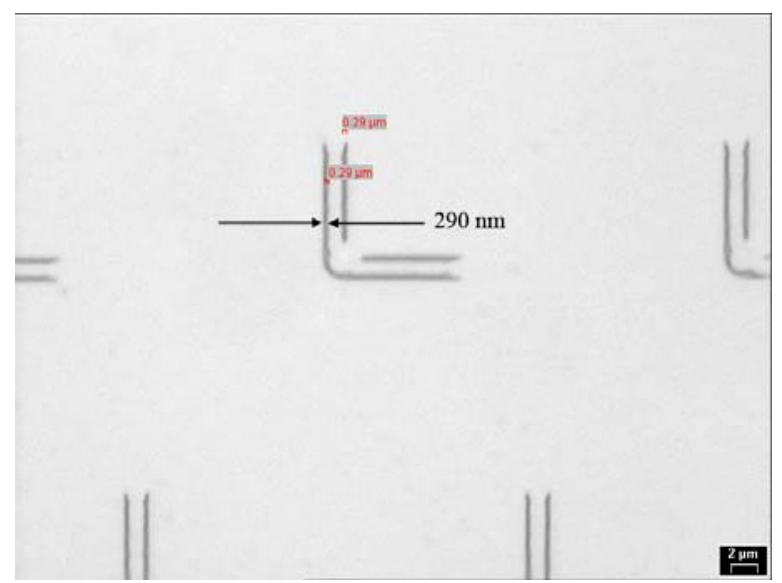

Fig. 4b. By using the fabricated phase shift structures (Fig. 4a) for UV exposure, periodic arrays of 'double L' were obtained.

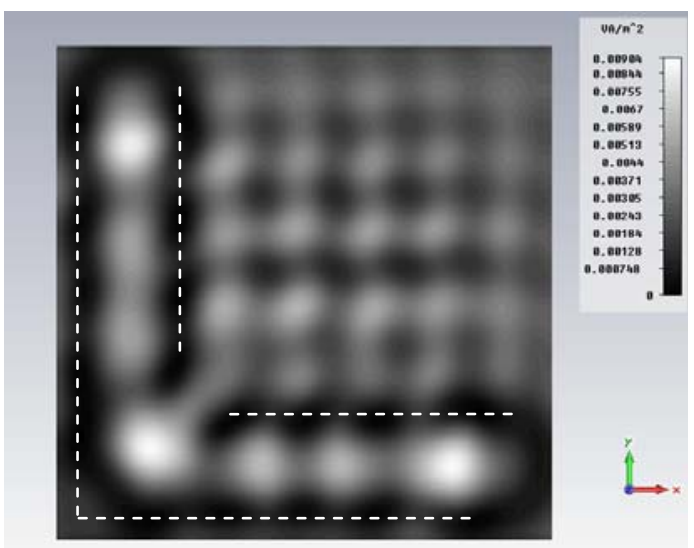

Fig. 4c. Simulated result showing the regions of intensity minimum at the edges of the structures.

In Fig. 5a, another design was adopted where an array of square phase shift structures were fabricated on fused silica. The interior edge is $\sim 2.85 \mu \mathrm{m}$ away from the outer edge. The 3D AFM image was included in the inset to illustrate the surface of the structure. It indicates the through hole in the centre of the square. Figure $5 \mathrm{~b}$ shows the patterns obtained by using the square phase shift structure for exposure. An array of 2 concentric squares 
patterns were obtained, with the smaller square inside the bigger square. Both the external and the internal edges of the square phase structure experience low light intensities due to destructive interference. This formed the 2 concentric square images on the photoresist. The exposure time can also be increased to get the patterns shown in Fig. 5c. Light entering the through hole at the centre of the square phase shift structure exposed the photoresist at the internal edge more easily than the photoresist at the external edge. Therefore, by increasing the exposure time, the interior square photoresist can be controlled to be exposed, leaving behind only the external square. This demonstrates the design flexibility available when the phase shift structures were used for nanopatterning.

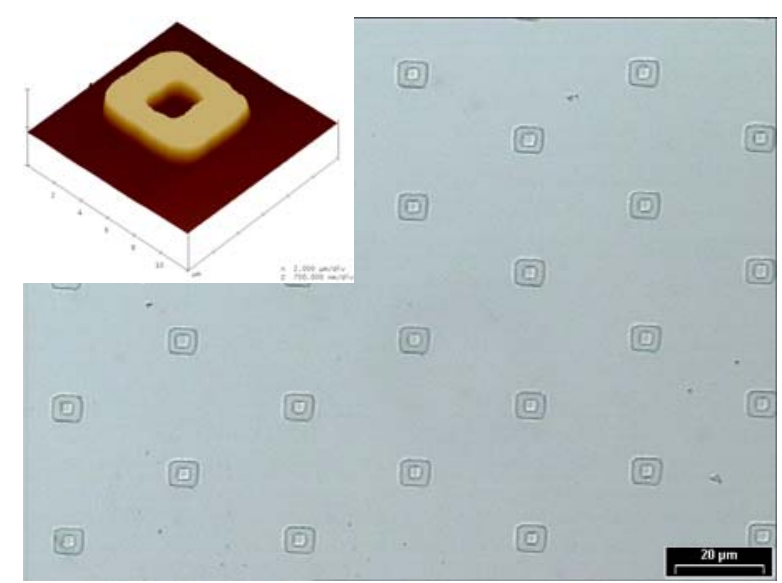

Fig. 5a. Square shaped phase shift structures array fabricated on fused silica. The inset shows the 3D AFM image, showing the through hole in the centre.

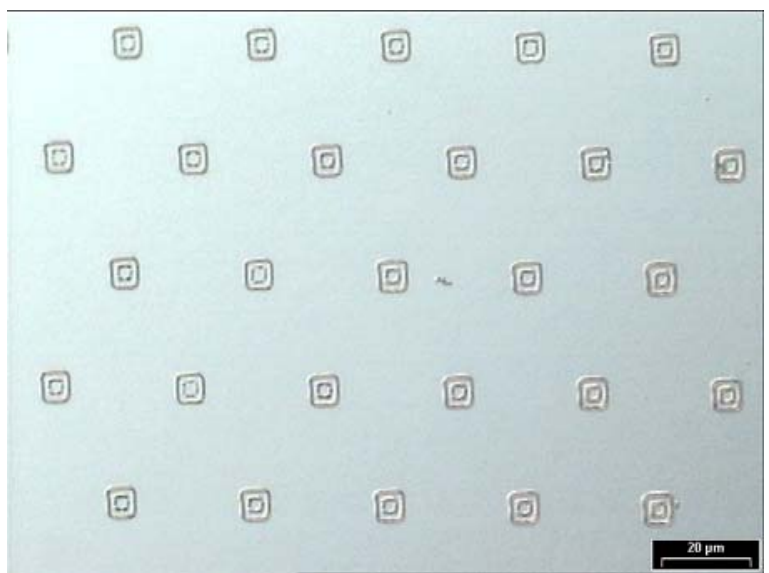

Fig. 5b. 2 concentric square patterns on photoresist. The smaller square is due to the internal edge of the square phase structure while the bigger square is due to the external edge.

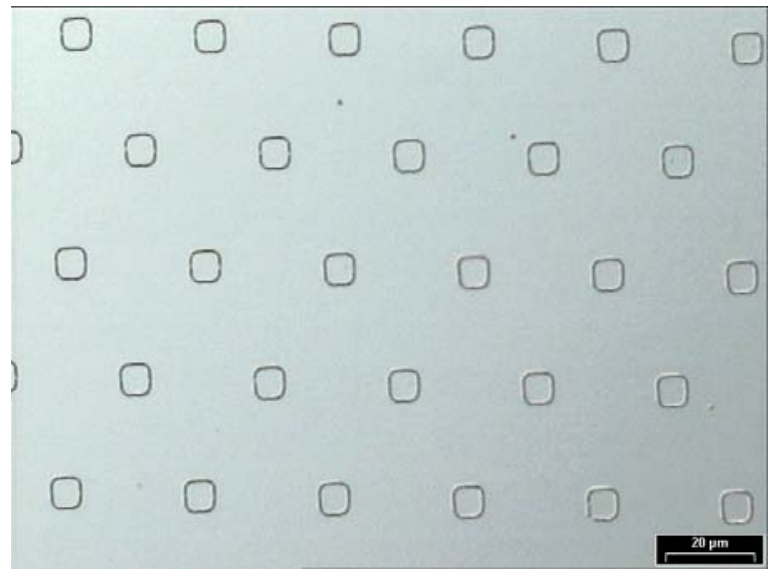

Fig. 5c By increasing the exposure time, the internal smaller square pattern on photoresist can be deliberately exposed, leaving behind only the external bigger square.

\section{Conclusions}

The laser MLA lithography technique was employed to pattern large array of periodic patterns on photoresist coated on fused silica substrate. The patterns were then transferred to the fused silica substrate to fabricate phase shift structures. The process flow for transferring the patterns to the $\mathrm{Cr}$ hard mask and then to the fused silica substrate was discussed. Subsequently, the fabricated phase shift structures were used as a mask for printing smaller feature sizes by making use of destructive interference of light that occurred at the edges of the structures. Lines with dimensions of $\sim 290 \mathrm{~nm}$ were printed and the results were verified with theoretical simulation. In this work, laser MLA lithography was demonstrated as a cost effective method for direct writing and fabrication of arbitrary structures on fused silica for phase shift mask lithography. It has the benefits of high throughput due to its parallel processing capabilities. The phase shift mask was then used successfully for patterning periodic array of nanostructures by UV light exposure.

\section{Acknowledgments}

Z. Q. Huang is grateful to GLOBALFOUNDRIES Singapore Pte Ltd for providing the scholarship, and Dr. Lap Chan and Dr. C. M. Ng for their guidance.

\section{References}

[1] T. F. Kosar, C. Chen, N. L. Stucky and A. Folch: J. Biomed. Nanotechnol., 1, (2005) 161.

[2] A. De Leebeeck, L. K. S. Kumar, V. de Lange, D. Sinton, R. Gordon and A. G. Brolo: Anal. Chem., 79, (2007) 4094.

[3] D. Falconnet, G. Csucs, H. M. Grandin and M. Textor: Biomaterials, 27 (2006) 3044.

[4] W. K. Choi, T. H. Liew, H. G. Chew, F. Zheng, C. V. Thompson, Y. Wang, M. H. Hong, X. D. Wang, L. Li and J. Yun: Small, 4, (2008) 330.

[5] J. Yun, R. Wang, W. K. Choi, J. T. L. Thong, C. V. Thompson, M. Zhu, Y. L. Foo and M. H. Hong: Carbon, 48, (2010) 1362.

[6] S. Grego, T. W. Jarvis, B. R. Stoner and J. S. Lewis: Langmuir, 21, (2005) 4971. 
[7] N. Liu, H. Guo, L. Fu, S. Kaiser, H. Schweizer and H. Giessen: Nat. Mater., 7, (2008) 31.

[8] V. M. Shalaev: Nat. Photonics, 1, (2007) 41.

[9] D. Schurig, J. J. Mock, B. J. Justice, S. A. Cummer, J. B. Pendry, A. F. Starr and D. R. Smith: Science, 314, (2006) 977.

[10]Z. C. Chen, M. H. Hong, C. S. Lim, N. R. Han, L. P. Shi and T. C. Chong: Appl. Phys. Lett., 96, (2010) 181101.

[11] Y. Lin, M. H. Hong, T. C. Chong, C. S. Lim, G. X. Chen, L. S. Tan, Z. B. Wang and L. P. Shi: Appl. Phys. Lett., 89, (2006) 041108.
[12] K. K. H. Toh, G. Dao, R. Singh and H. Gaw: Proc. SPIE, 1496, (1991) 27.

[13] L. Bauch, J. Bauer, H. Dreger, B. Lauche, G. Mehlib and St. Rothe: Microelectron. Eng., 17, (1992) 87.

[14] J. F. Chen, J. S. Petersen, R. Socha, T. Laidig, K. E. Wampler, K. Nakagawa, G. Hughes, S. MacDonald and W. Ng: Proc. SPIE, 4346, (2001) 515.

[15] M. Fritze, J. M. Burns, P. W. Wyatt, D. K. Astolfi, T. Forte, D. Yost, P. Davis, A. V. Curtis, D. M. Preble, S. Cann, S. Denault, H. Y. Liu, J. C. Shaw, N. T. Sullivan, R. Brandom and M. E. Mastovich: Proc. SPIE, 4000, (200 0) 388 .

(Received: June 7 , 2010, Accepted: November 01, 2010) 